\title{
Fishes from Complex A offerings of Templo Mayor of Tenochtitlan (Mexico City, Mexico)
}

\author{
ANA FABIOLA GUZMÁN \\ Subdirección de laboratorios y Apoyo Académico, Instituto Nacional de Antropología e Historia, Moneda 16, Col. \\ Centro, Ciudad de México, CP 06060, México, \\ e-mail: ana_guzman@inah.gob.mx, \\ and
}

Escuela Nacional de Ciencias Biológicas, Instituto Politécnico Nacional, Prolongación de Carpio y Plan de Ayala, Ciudad de México, CP 11340, México.

(Received 1 September 2016; Revised 25 January 2017; Accepted 10 February 2017)

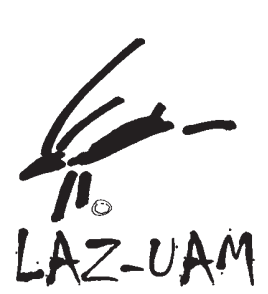

RESUMEN: El presente trabajo aborda los restos de peces encontrados en las once ofrendas que integran el Complejo A, depositadas alrededor del Templo Mayor de Tenochtitlán, el cual fuera el principal edificio ceremonial de la cultura mexica. El estudio de más de 35,000 restos muestra que se usaron 391 individuos de 63 especies y 35 familias. Siete especies fueron particularmente importantes por la cantidad de individuos y su frecuencia de uso, aunque el único atributo en común a las 11 ofrendas, es el pez sierra (Pristis). Sólo fueron ofrendados peces marinos, predominando las formas del Atlántico. La mayoría de los peces fueron depositados con una preparación taxidérmica para eliminar la columna vertebral. Las ofrendas colocadas en el relleno constructivo fueron marcadamente menos diversas respecto de las otras ofrendas colocadas en cistas. Aunque se pudieron relacionar algunas especies mencionadas por Sahagún con las ofrendadas, las fuentes etnohistóricas proporcionan poca información de los peces y su uso, de ahí la importancia de un apropiado rescate y estudio de los restos de peces como fuente primaria y casi exclusiva de las relaciones entre el hombre mesoamericano y este recurso.

PALABRAS CLAVE: TEMPLO MAYOR, MEXICA, OFRENDAS, PECES, MÉXICO

ABSTRACT: This paper deals with the fish remains found in the 11 offerings known as Complex A, deposited around the Templo Mayor of Tenochtitlan, the main ceremonial building of the Mexica culture. The study of over 35,000 fish remains showed that 391 individuals from 63 species and 35 families were present. Seven species were particularly important because of the number of individuals and their frequency, although the only fish found in all the offerings was the sawfish (Pristis). Only marine species were included in the offerings, most of which came from the Atlantic Ocean. Many of the fish had a taxidermic preparation of one kind or another. The offerings placed directly in the fill were noticeably less diverse compared to the offerings placed in ashlar stone boxes. Although some of the offered species were related to the ones mentioned by the Aztec historian Sahagún, ethnohistorical sources provide little information about marine fish and their ritual use; hence the importance of proper recovery and study of those remains as the primary and almost exclusive source of the relationship between the Mexica people and this faunal resource.

KEYWORDS: TEMPLO MAYOR, MEXICA, OFFERINGS, FISHES, MÉXICO 


\section{INTRODUCCIÓN}

The discovery of the archaeological site known as Templo Mayor of Tenochtitlan happened in February, 1978, during a salvage operation (García Cook \& Arana, 1978). As soon as the archaeologists realized they had located the foundations of the long-lost and much search-for main temple of the Mexica culture, the salvage procedure was replaced by a research project (Matos Moctezuma, 1990). This finding allowed the historical documents, previously the main source of information about the site, to be validated. While the excavations advanced, archaeologists became aware, first, that under the preserved floors and platforms there were a lot of still-intact offerings, at present count 153 (López Luján \& Chávez Balderas, 2010). Second, many of these offerings contained enormous quantities of animal remains, an unprecedented event that deserved a special archaeozoological project following four main research lines (Polaco, 1991).

Several researchers have studied the faunal remains since 1978. The fishes, however, were difficult to analyze because of the fish osteology itself and the offering's complexity, so the initial studies were based only on the most diagnostic materials (Díaz-Pardo, 1982; Carramiñana A., 1988; Díaz-Pardo \& Teniente-Nivón, 1991). In time, a restudy of the fish remains of some of the offerings excavated early in the project was done, revealing relevant changes which stimulated a continued analysis (Guzmán \& Polaco, 1999, 2000, 2003). The present study provides an updated inventory of the fish contained in the 11 offerings known as Complex A, excavated between 1978 and 1982 by the Templo Mayor Project archaeologists, and a comparison of the fish content among the offerings and with ethnohistorical data is also provided.

\section{Cultural and historical context}

Mexica people were the last human group to settle in the densely populated basin of Mexico. They founded the city of Mexico-Tenochtitlan on an island at Lake Anáhuac in A.D. 1325, with the permission of the Azcapotzalca people, to whom Mexica paid tribute. This situation was gradually reversed, and 200 years later, in A.D. 1519 when Spaniards arrived in the current Mexican territory, the Mexica Empire dominated a vast region inside and outside the basin, expanding toward the Atlantic and Pacific coasts.

The Templo Mayor was the main ceremonial Mexica building and was enlarged and renovated by each new ruler. It had a particular orientation and construction symbolizing the order of the Mesoamerican universe. The temple was devoted to two gods, Tláloc, in the northern half of the temple, and Huitzilopochtli, in the southern half; the latter was also the patron god of the Mexica (López Luján, 1993).

There is little ethnohistorical information about the offerings within the Templo Mayor, therefore this contexts are a fresh source of information about Mexica ceremonial customs, especially because they occur in almost undisturbed discrete units with an orderly content that should reflect of the symbolic language of the site (López Luján, 1993). The animals offered, by extension, ought to be part of the oblation language.

\section{Complex a offerings}

A 1993-analysis of the similarities among the offerings defined several clusters or offerings complexes. Complex A included the 11 richest-in-objects offerings (Offerings 1, 6, 7, 11, 13, 17, 20, 23, 60, 61 and 88) that had fish as one of their most relevant attributes (Figure 1a; López Luján, 1993). However, for unspecified reasons, the biological contents of the offerings were given unequal treatment: species of mammals and birds were itemized, whereas other animals such as fish were handled as a group.

Complex A offerings were placed around the building (Figure 1b) supposedly during its consecration ceremony, between A.D. 1469 and 1481 (López Luján, 1993), more than 100 years after the city was founded. At that time, Mexica had gained independence from Azcapotzalco and their territorial expansion had begun.

Except for Offering 60, the offerings were located on the main axes of symmetry of the building, with three of the four pairs of similar offerings opposite to each other in most cases (Figure 1b; López Luján, 1993).

Offerings 23, 61 and 88 were on the Tláloc side, Offerings 1, 6, 7 and 60 were on the Huitzilopochtli side and Offerings $11,13,17$, and 20 were on 
a) Complex A cluster (detail of general dendrogram)

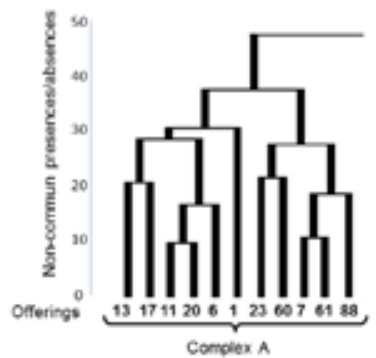

b) Location of Complex A offerings around the Templo Mayor: excavation plan

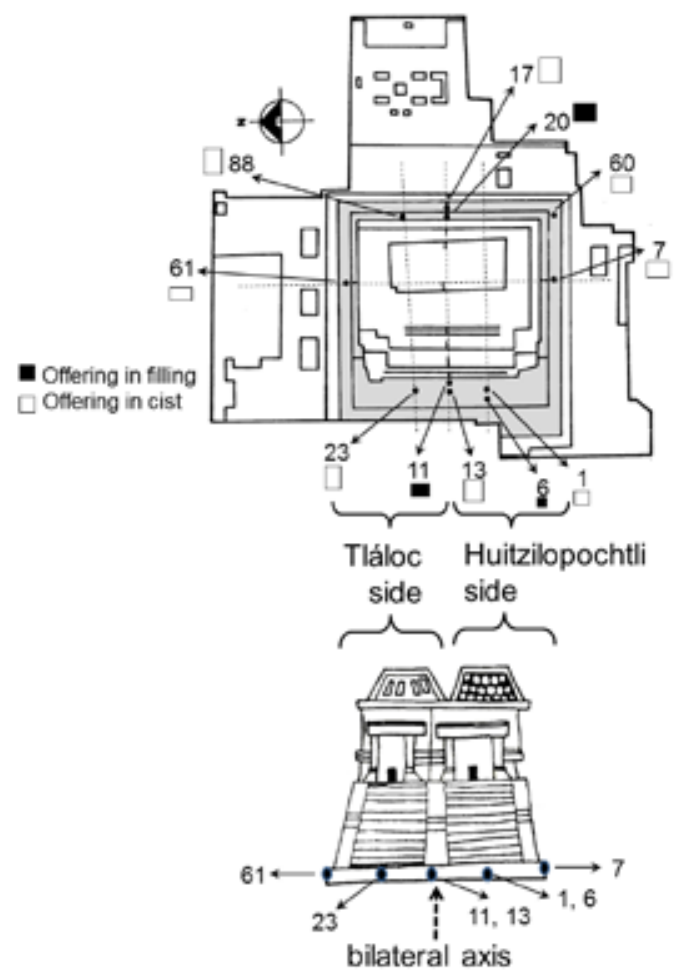

c) Location of Complex A offerings around the Templo Mayor: building according to Sahagún.

FIGURE 1

Clusters and location of Complex A offerings around the Templo Mayor. Taken and modified from López Luján (1993), including the figure of the Templo Mayor from Sahagún (Primeros memoriales).

the bilateral axis dividing the Tláloc and Huitzilopochtli halves of the temple (Figures $1 \mathrm{~b}$ and 1c). Five of the offerings were on the main façade (1, $6,11,13$, and 23$)$, three on the rear $(17,20$, and 88 ), two on the lateral sides (7, and 61), and one was on a rear corner (60). Offerings 6,11 and 20, located on the bilateral axis and on Huitzilopochtli side, were placed directly on the original ground surface (referred to here as the fill). The remaining offerings were isolated from the fill in ashlar stone boxes or cists.

\section{MATERIAL AND METHODS}

An exhaustive search of all the fish remains was performed (the bones had been dispersed for sev- 
eral reasons), both in the facilities of the Templo Mayor Museum and in the Laboratory of Archaeozoology “M. en C. Ticul Álvarez Solórzano". The remains were analyzed according to conventional archaeozoological methods, and included the anatomical and taxonomic identification, the quantification of remains and of individuals (minimal number of individuals $[M N I]$ or abundance), the form fish was offered and zones from which the fish were procured. To document fish procurement areas, several ichthyologic publications were consulted (e.g., Allen \& Robertson, 1994; Humann, 1994; Castro-Aguirre et al., 1999; Bedia Sánchez \& Franco López, 2008; besides personal field work).

The exhaustive search and identification procedures carried out allowed to consider each offering content as a "community" and to characterize it using ecological descriptors, such as richness, abundance, frequency, importance value index (IVI), diversity $\left(H^{\prime}\right.$, or Shannon-Wierner index), dominance $\left(D\right.$, or Simpson index), equitability $\left(J^{\prime}\right)$; to correlate the offerings'contents to location and type of container (Spearman's rho); and to perform similarity analysis based on cluster and ordination (PCA) methods using presence/absence (Jaccard and simple matching coefficients) and abundance (Morisita coefficient) data. In this paper, the results of the analysis are presented to the species identification level, which may differ from the results obtained using other taxonomic categories (Guzmán \& Polaco, 2003; Guzmán, 2007).

A more detailed description on the archaeozoological and ecological techniques employed can be found at Crisci \& López-Armengol (1983), Klein \& Cruz-Uribe (1984), Reitz \& Wing (1999), Guzmán \& Polaco (2000), Smith \& Smith (2004), and Guzmán (2007), among other works.

\section{RESULTS AND DISCUSSION}

\section{Faunal composition}

The study is based on more than 35,000 remains from 391 individuals, 35 families and 63 species, of which nine species are sharks, rays, and sawfish and the remainder, are bony fishes (Table 1). Thirty times more materials were examined than in the previous study (vs. 1154 remains: Díaz-Pardo, 1982; Carramiñana A., 1988; Díaz-Pardo \& Teniente
Nivón, 1991), which resulted in almost doubling the number of species (vs. 36), although the number of individuals changed little (vs. 367). The diversity of the overall assemblage is one of the highest recorded for an inland Mexican archaeological site $\left(H^{\prime}=\right.$ 4.91) (for a general comparison of the archaeoichthyological record, see Polaco \& Guzmán, 1997).

More than half of the species were documented in the Mexican archaeological record for the first time [Table 1. For a comparison see Polaco \& Guzmán (1997), as well as recent archaeoichthyological studies, e.g., Kennett et al. (2008), Rodríguez Galicia \& Valadez Azúa (2013)]. Fish commonly found in modern markets and in Mexican archaeoichthyological collections, especially from domestic contexts, such as sea catfish (Ariidae), snook (Centropomidae) and croakers (Sciaenidae) (Polaco \& Guzmán, 1997), are noteworthy for their absence among these offerings. This absence could be related to the exclusive ceremonial role of the Templo Mayor.

Only seven species dominate the Complex A offerings (Table 2: $I V I>6$ ). These are ballyhoo and halfbeak (Hemiramphus brasiliensis and Hyporhamphus sp.), houndfish (Tylosurus crocodilus), Spanish hogfish (Bodianus cf. B. rufus), French angelfish (Pomacanthus paru), porcupinefish (Diodon hystrix) and sawfish (Pristis pectinata and Pristis sp.). Sawfish (Pristis) is the only taxon common to the eleven offerings, although not many individuals were used. These seven species, due to their frequency and abundance, could be then interpreted as basic components of the language of these offerings.

\section{Fish procurement areas}

All the specimens are marine fishes. The material reported earlier in Offering 1 as freshwater silverside (family Atherinopsidae) by Carramiñana A. (1988), was not located but instead remains of Hemiramphidae were present; therefore, there was probably a mistaken attribution as the skull roof is very similar in both animals. Species from the Atlantic Ocean (Gulf of Mexico and Caribbean Sea) predominate (33) and only six species are from the Pacific Ocean (Table 1: Negaprion fronto, Lutjanus ca. L. argentiventris, Scarus perrico, Prionurus punctatus, Arothron sp., and Sphoeroides annulatus). An additional group is undetermined because 


\begin{tabular}{|c|c|c|c|c|}
\hline $\begin{array}{l}\text { Numbers account the } 6 \\
\text { nate, present in both oc } \\
\mathrm{R}=\text { reefs, } \mathrm{W}=\text { several }\end{array}$ & $\begin{array}{l}\text { t species. Coast of provenance: } \mathrm{A}=\mathrm{Atla} \\
\text { ndeterminate. Environment: } \mathrm{L}=\text { littoral, } \\
\text { dd reef environments, } \mathrm{Ws}=\text { several envi }\end{array}$ & $\begin{array}{l}\text { Ocean }(\text { Gulf of Mex } \\
=\text { littoral and oceanic } \\
\text { ments and stenohalin }\end{array}$ & $\begin{array}{l}\text { n Sea), } P=\text { Pacific Oc } \\
\text { stenohaline, Lu = littc } \\
\text { nvironments and eury }\end{array}$ & $\begin{array}{l}\mathrm{n}, \mathrm{B}=\text { indetermi- } \\
1 \text { and euryhaline } \\
\text { line }\end{array}$ \\
\hline Class & Species & Common Name & Coast of providence & Environment \\
\hline Order & & & & \\
\hline Family & & & & \\
\hline Carcharhiniformes & & & & \\
\hline Carcharhinidae & 1. Carcharhinus leucas & Bull shark & $\mathrm{B}$ & $\mathrm{Lu}$ \\
\hline & 2. $\quad$ Carcharhinus limbatus & Blacktip shark & $\mathrm{B}$ & Ws \\
\hline & Carcharhinus sp. & & I & $\mathrm{W}$ \\
\hline & 3. Galeocerdo cuvier & Tiger shark & $\mathrm{B}$ & $\mathrm{Wu}$ \\
\hline & 4. * Negaprion fronto & Lemon shark & $\mathrm{P}$ & Ws \\
\hline & Negaprion sp. & & I & Ws \\
\hline Sphyrnidae & & & & \\
\hline & 5. Sphyrna mokarran & Great hammerhead & B & Ws \\
\hline Rajiformes & & & & \\
\hline Pristidae & 6. $\quad$ Pristis pectinata & Smalltooth sawfish & $\mathrm{B}$ & $\mathrm{Lu}$ \\
\hline & Pristis sp. & & I & $\mathrm{Lu}$ \\
\hline Dasyatidae & 7. $\quad$ Dasyatis sp. & Stingray & $\mathrm{I}$ & W \\
\hline & 8. * Himantura sp. & Stingray & $\mathrm{I}$ & $\mathrm{L}$ \\
\hline & Dasyatidae gen.et sp. indet. & & $\mathrm{I}$ & $\mathrm{L}$ \\
\hline Myliobatidae & 9. $\quad$ Aetobatus narinari & Spotted eagle ray & $\mathrm{B}$ & Ws \\
\hline Actinopterygii & & & & \\
\hline Clupeiformes & & & & \\
\hline Clupeidae & 10. Clupeidae gen. et sp. indet. & Shad, sardine & $\mathrm{I}$ & $\mathrm{L}$ \\
\hline Batrachoidiformes & & & & \\
\hline Batrachoididae & 11. Opsanus sp. & Toadfish & $\mathrm{A}$ & $\mathrm{W}$ \\
\hline Lophiiformes & & & & \\
\hline Ogcocephalidae & 12. * Ogcocephalus sp. & Batfish & $\mathrm{A}$ & Ws \\
\hline Beloniformes & & & & \\
\hline Belonidae & 13. * Ablennes hians & Flat needlefish & $\mathrm{B}$ & Lo \\
\hline & 14. * Strongylura marina & Atlantic needlefish & $\mathrm{A}$ & $\mathrm{Wu}$ \\
\hline & 15. * Strongylura cf. S. timиси & Redfin needlefish & $\mathrm{A}$ & $\mathrm{Wu}$ \\
\hline & Strongylura sp. & & $\mathrm{I}$ & $\mathrm{Wu}$ \\
\hline & 16. * Tylosurus crocodilus & Houndfish & $\mathrm{B}$ & Ws \\
\hline Exocoetidae & 17. * Exocoetidae gen. et $s p$. indet. & Flyingfish & $\mathrm{I}$ & Lo \\
\hline Hemirhamphidae & 18. * Hemirhamphus brasiliensis & Ballyhoo & $\mathrm{A}$ & Ws \\
\hline & 19. * Hyporhamphus sp. & Halfbeak & $\mathrm{I}$ & $\mathrm{L}$ \\
\hline Beryciformes & & & & \\
\hline Holocentridae & 20. * Holocentrus sp. & Squirrelfish & $\mathrm{A}$ & $\mathrm{R}$ \\
\hline Gasterosteiformes & & & & \\
\hline Fistulariidae & 21. * Fistularia $\mathrm{sp.}$ & Cornetfish & $\mathrm{I}$ & $\mathrm{R}$ \\
\hline Scorpaeniformes & & & & \\
\hline Dactylopteridae & 22. * Dactylopterus volitans & Flying gurnard & $\mathrm{A}$ & Ws \\
\hline Scorpaenidae & 23. * Scorpaena sp. & Scorpionfish & $\mathrm{I}$ & $\mathrm{W}$ \\
\hline Triglidae & 24. * Prionotus tribulus & Bighead searobin & A & Ls \\
\hline & * Prionotus sp. & & $\mathrm{I}$ & Ls \\
\hline Perciformes & & & & \\
\hline Serranidae & 25. * Epinephelus cf. E. adscensionis & Rock hind & $\mathrm{A}$ & $\mathrm{Wu}$ \\
\hline & Epinephelus sp. & & $\mathrm{I}$ & $\mathrm{W}$ \\
\hline Carangidae & 26. Caranx hippos & Crevalle jack & A & $\mathrm{Lu}$ \\
\hline & 27. * Oligoplites cf. O. saurus & Leatherjack & A & $\mathrm{Lu}$ \\
\hline & 28. * Selene cf. S. vomer & Lookdown & A & $\mathrm{Lu}$ \\
\hline Lutjanidae & 29. * Lutjanus cf. L. analis & Mutton snapper & $\mathrm{A}$ & $\mathrm{Wu}$ \\
\hline
\end{tabular}




\begin{tabular}{|c|c|c|c|c|}
\hline \multicolumn{5}{|l|}{ Tabla 1. (continuated) } \\
\hline & 30. Lutjanus ca. L. argentiventris & \begin{tabular}{|l|} 
Yellow snapper \\
\end{tabular} & $\mathrm{P}$ & Ws \\
\hline & 31. Lutjanus cf. L. griseus & Gray snapper & A & $\mathrm{Wu}$ \\
\hline & Lutjanus argentiventris/L.griseus & Snapper & I & \\
\hline & 32. Ocyurus chrysuru & Yellowtail snapper & A & $\mathrm{R}$ \\
\hline Lobotidae & 33. Lobotes surinamensis & Tripletail & B & $\mathrm{Lu}$ \\
\hline \multirow[t]{4}{*}{ Haemulidae } & 34. * Anisotremus surinamensis & Black margate & A & $\mathrm{R}$ \\
\hline & 35. * Anisotremus virginicus & Porkfish & A & $\mathrm{R}$ \\
\hline & 36. * Haemulon cf. H. carbonarium & Caesar grunt & A & $\mathrm{R}$ \\
\hline & 37. * Haemulon flavolineatum & French grunt & A & Ws \\
\hline Pomacanthidae & 38. * Pomacanthus paru & French angelfish & A & $\mathrm{R}$ \\
\hline Kyphosidae & 39. * Kyphosus sp. & Chub & $\mathrm{I}$ & $\mathrm{R}$ \\
\hline \multirow[t]{2}{*}{ Pomacentridae } & 40. * Abudefduf sp. & Damselfish & I & $\mathrm{R}$ \\
\hline & 41. * Microspathodon sp. & Yellowtail damselfish & I & $\mathrm{R}$ \\
\hline \multirow[t]{2}{*}{ Labridae } & 42. Bodianus cf. B. rufus & Spanish hogfish & A & $\mathrm{R}$ \\
\hline & 43. * Halichoeres radiatus & Puddingwife & A & $\mathrm{R}$ \\
\hline \multirow[t]{5}{*}{ Scaridae } & 44. Scarus perrico & Bumphead parrotfish & $\mathrm{P}$ & $\mathrm{R}$ \\
\hline & 45. * Scarus vetula & Queen parrotfis & A & $\mathrm{R}$ \\
\hline & 46. * Sparisoma cf. S. aurofrenatum & Redband parrotfish & A & $\mathrm{R}$ \\
\hline & 47. * Sparisoma rubripinne & Redfin parrotfish & A & $\mathrm{R}$ \\
\hline & 48. Sparisoma viride & Stoplight parrotfish & A & $\mathrm{R}$ \\
\hline Ephippidae & 49. * Chaetodipterus faber & Spadefish & A & $\mathrm{Wu}$ \\
\hline \multirow[t]{2}{*}{ Acanthuridae } & 50. Acanthurus sp. & Surgeonfish & I & $\mathrm{R}$ \\
\hline & 51. Prionurus punctatus & Yellowtail surgeonfish & $\mathrm{P}$ & $\mathrm{R}$ \\
\hline Sphyraenidae & 52. Sphyraena barracuda & Great barracuda & A & Ws \\
\hline Trichiuridae & 53. * Trichiurus lepturus & Cutlassfish & A & Ls \\
\hline \multicolumn{5}{|l|}{ Tetraodontiformes } \\
\hline Balistidae & 54. * Canthidermis sufflamen & Ocean triggerfish & A & $\mathrm{R}$ \\
\hline \multirow[t]{2}{*}{ Monacanthidae } & 55. * Aluterus sp. & Leatherjacket & I & $\mathrm{W}$ \\
\hline & 56. * Cantherhines sp. & Filefish & I & $\mathrm{R}$ \\
\hline \multirow[t]{3}{*}{ Ostraciidae } & 57. * Acanthostracion sp. & Cowfish & A & $\mathrm{Wu}$ \\
\hline & 58. * Lactophrys triqueter & Smooth trunkfish & A & $\mathrm{R}$ \\
\hline & Ostraciidae gen. et sp. indet. & & I & $\mathrm{W}$ \\
\hline \multirow[t]{2}{*}{ Tetraodontidae } & 59. * Arothron sp. & Puffer & $\mathrm{P}$ & $\mathrm{R}$ \\
\hline & 60. * Sphoeroides annulatus & Bullseye puffer & $\mathrm{P}$ & $\mathrm{Wu}$ \\
\hline \multirow[t]{4}{*}{ Diodontidae } & 61. * Chilomycterus schoepfi & Stripped burrfish & A & $\mathrm{Wu}$ \\
\hline & 62. * Diodon holacanthus & Balloonfish & B & $\mathrm{R}$ \\
\hline & 63. Diodon hystrix & Porcupinefish & B & Ws \\
\hline & Diodon sp. & & $\mathrm{B}$ & \\
\hline
\end{tabular}

TABLE 1

Fishes Identified in Complex A Offerings of Templo Mayor of Tenochtitlan.

the taxa could inform either coast (e.g. Tylosurus crocodylus and Acanthurus sp.).

The composition of species indicate that three primarily environments were exploited. Reefs were one of these, with a set of fishes exclusive from such areas. The second group inhabits the coastal marine environment but rarely travels to the reefs, and the third set has a wider ecological distribution (Table 1). Thus, most fishes were obtained from a platform near the reefs, possibly using poisons and hooks in the reef area and nets in more open, sandy parts, techniques already known by that time (Guzmán \& Polaco, 2007). Fishes could have also been obtained by collecting them dead at the beach during red tide events that regularly affect the Mexican coasts (Sevilla, 1977; Díaz-Pardo \& Teniente-Nivón, 1991; pers. obs.). The closest areas matching this type of mixed environment are located over $300 \mathrm{~km}$ from Templo Mayor, in the states of Veracruz (the Veracruz Coral Reef System) on the Atlantic slope, and the rocky coasts of Guerrero and Oaxaca, on the Pacific coast (Carricart-Ganivet \& Horta-Puga, 1993; Reyes Bonilla, 1993). 
$\mathrm{MNI}=$ minimum number of individuals. $\mathrm{NO}=$ number of offerings containing the species.

\begin{tabular}{|c|c|c|c|c|c|c|c|}
\hline Species & MNI & $\mathrm{NO}$ & IVI & Species & MNI & NO & IVI \\
\hline Hyporhamphus sp. & 66 & 7 & 21.15 & Diodon sp. & 3 & 1 & 1.38 \\
\hline Tylosurus crocodilus & 48 & 7 & 16.54 & Selene cf. S. vomer & 2 & 1 & 1.12 \\
\hline Bodianus cf. B. rufus. & 27 & 5 & 9.95 & Lutjanus ca. L. argentiventris & 2 & 1 & 1.12 \\
\hline Pristis pectinata & 17 & 9 & 9.84 & Lutjanus cf. L. griseus & 2 & 1 & 1.12 \\
\hline Pomacanthus paru & 22 & 6 & 9.29 & Ocyurus chrysurus & 2 & 1 & 1.12 \\
\hline Diodon hystrix & 20 & 6 & 8.77 & Carcharhinus sp. & 1 & 1 & 0.87 \\
\hline Hemiramphus brasiliensis & 15 & 8 & 8.71 & Galeocerdo cuvier & 1 & 1 & 0.87 \\
\hline Pristis sp. & 12 & 8 & 7.95 & Negaprion fronto & 1 & 1 & 0.87 \\
\hline Exocoetidae gen. et sp. indet. & 9 & 5 & 5.35 & Negaprion sp. & 1 & 1 & 0.87 \\
\hline Anisotremus virginicus & 12 & 4 & 5.51 & Sphyrna mokarran & 1 & 1 & 0.87 \\
\hline Carcharhinus leucas & 10 & 3 & 4.39 & Aetobatus narinari & 1 & 1 & 0.87 \\
\hline Strongylura sp. & 9 & 3 & 4.13 & Clupeidae gen. et sp. indet. & 1 & 1 & 0.87 \\
\hline Epinephelus cf. E. adscensionis & 8 & 3 & 3.88 & Ablennes hians & 1 & 1 & 0.87 \\
\hline Carcharhinus limbatus & 5 & 4 & 3.72 & Strongylura cf. S. timиси & 1 & 1 & 0.87 \\
\hline Canthidermis sufflamen & 5 & 4 & 3.72 & Holocentrus sp. & 1 & 1 & 0.87 \\
\hline Halichoeres radiatus & 5 & 3 & 3.11 & Fistularia sp. & 1 & 1 & 0.87 \\
\hline Chilomycterus schoepfii & 4 & 3 & 2.85 & Epinephelus sp. & 1 & 1 & 0.87 \\
\hline Dasyatidae gen. et sp. indet. & 3 & 3 & 2.60 & Lutjanus cf. L. analis & 1 & 1 & 0.87 \\
\hline Opsanus sp. & 3 & 3 & 2.60 & Lutjanus argentiventris/L. griseus & 1 & 1 & 0.87 \\
\hline Haemulon flavolineatum & 3 & 3 & 2.60 & Lobotes surinamensis & 1 & 1 & 0.87 \\
\hline Sphyraena barracuda & 5 & 2 & 2.50 & Anisotremus surinamensis & 1 & 1 & 0.87 \\
\hline Diodon holacanthus & 5 & 2 & 2.50 & Haemulon cf. H. carbonarium & 1 & 1 & 0.87 \\
\hline Caranx hippos & 3 & 2 & 1.99 & Microspathodon sp. & 1 & 1 & 0.87 \\
\hline Sparisoma rubripinne & 3 & 2 & 1.99 & Abudefduf sp. & 1 & 1 & 0.87 \\
\hline Dasyatis sp. & 2 & 2 & 1.73 & Scarus perrico & 1 & 1 & 0.87 \\
\hline Himantura sp. & 2 & 2 & 1.73 & Scarus vetula & 1 & 1 & 0.87 \\
\hline Strongylura marina & 2 & 2 & 1.73 & Sparisoma cf. S. aurofrenatum & 1 & 1 & 0.87 \\
\hline Dactylopterus volitans & 2 & 2 & 1.73 & Chaetodipterus faber & 1 & 1 & 0.87 \\
\hline Scorpaena sp. & 2 & 2 & 1.73 & Prionurus punctatus & 1 & 1 & 0.87 \\
\hline Prionotus sp. & 2 & 2 & 1.73 & Trichiurus lepturus & 1 & 1 & 0.87 \\
\hline Kyphosus sp. & 2 & 2 & 1.73 & Aluterus sp. & 1 & 1 & 0.87 \\
\hline Sparisoma viride & 2 & 2 & 1.73 & Cantherhines sp. & 1 & 1 & 0.87 \\
\hline Acanthurus sp. & 2 & 2 & 1.73 & Acanthostracion sp. & 1 & 1 & 0.87 \\
\hline Arothron sp. & 2 & 2 & 1.73 & Lactophrys triqueter & 1 & 1 & 0.87 \\
\hline Prionotus tribulus & 4 & 1 & 1.63 & Ostraciidae no identificado & 1 & 1 & 0.87 \\
\hline Ogcocephalus sp. & 3 & 1 & 1.38 & Sphoeroides annulatus & 1 & 1 & 0.87 \\
\hline
\end{tabular}

TABLE 2

Importance Value Index of Fish Species from Complex A Offerings.

\section{Body parts offered up}

The body parts represented as well as the cut marks provided insights on how fish were offered. In particular, cut marks were recorded in more than 100 bones from 41 individuals, 20 species and 8 different offerings (Figure 2a). All this showed a variety of treatments of the fish body. Many fishes had a taxidermy preparation that exclusively extracted the backbone (except the tail and fins) making the fish look like as though they were still intact (Figure 2b). To a lesser degree some were offered up as whole specimens. In other cases, just the anterior third of the fish, the jaws of sharks and rays, sawfish rostrums and some headless specimens were offered. Offering whole, or apparently whole, fish was the common pattern, except for the offerings placed onto the fill (Offerings 6, 11, 20), which contained mainly sawfish rostra, and isolated teeth, vertebra, and dermal structures. 
As the preference was to offer fishes that appeared complete, it is possible that the choice was related to the appearance of each species, as explained in previous works (Díaz-Pardo \& Teniente-Nivon, 1991; Guzmán \& Polaco, 1999, 2000). The color pattern may have played a decisive role because some species present facial masks (e.g., Anisotremus virginianus, Chaetodipterus faber, Ocyurus chrysurus, etc.) in the same way Mexica gods did (as Xipe Tótec, Tláloc and Tezcatlipoca). These fish are not usually the most important ones, thus their participation in the language of the offerings might be the representation of a god or an idea, particularizing the offering message in this way.
Fish species comparison among offerings

As for the number of species and individuals, bilateral axis offerings were comparatively poorer, especially the ones deposited in the fill, while the offerings dedicated to Huitzilopochtli and Tláloc were richer, especially Offerings 23 and 7, the first one located on the façade and the latter on the south side (Table 3). Certainly, these observations are reflected by the ecological indices: diversity $\left(H^{\prime}\right)$ and equitability $\left(J^{\prime}\right)$ are particularly low in the bilateral axis offerings while they are high for the offerings from the main shrines, except for Offering 6 which

a) Material with cut-marks

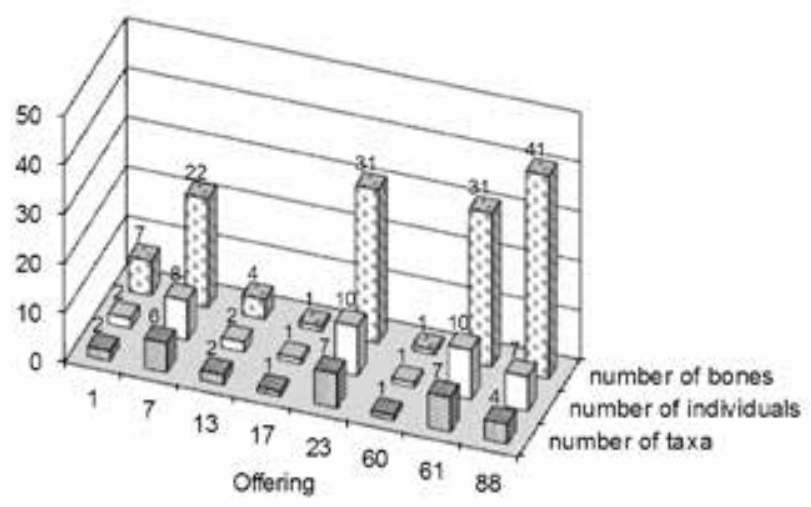

b) Body parts offered up

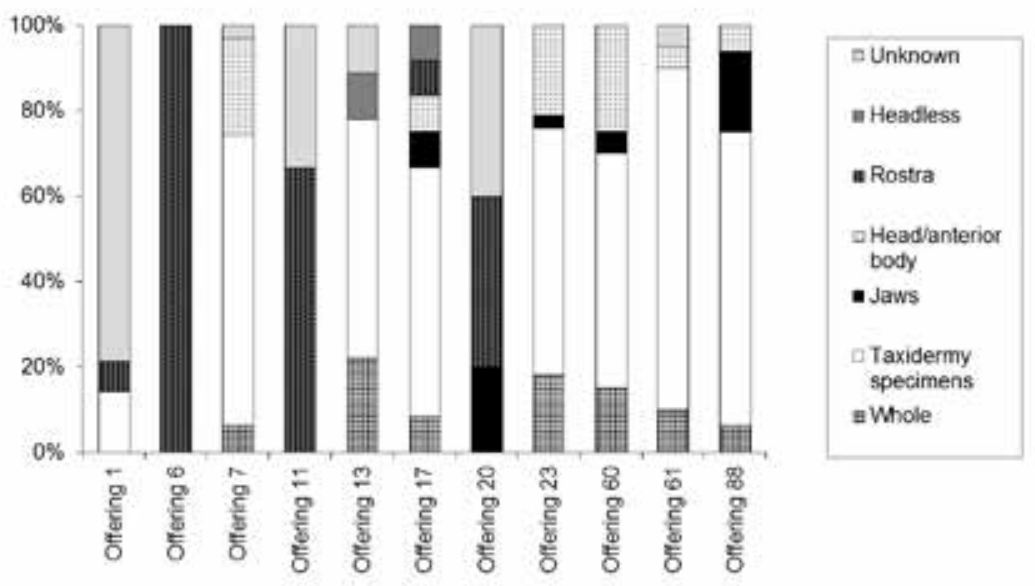

FIGURE 2

Types of body preparation for fishes in the Complex A offerings. 
Cluster analysis $\quad$ Principal component analysis

a) presence/absence
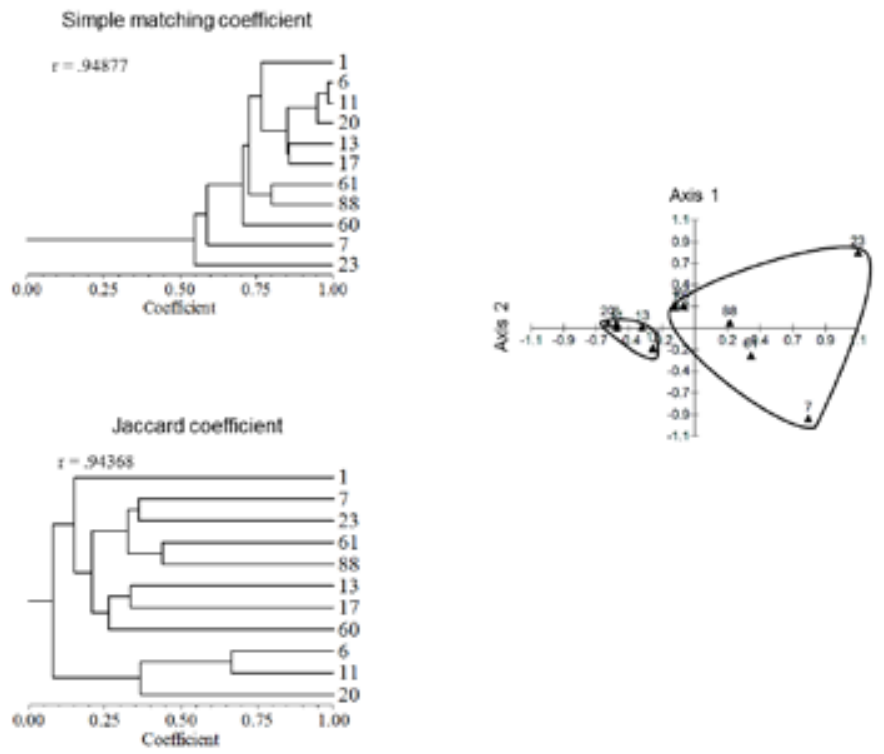

b) abundance
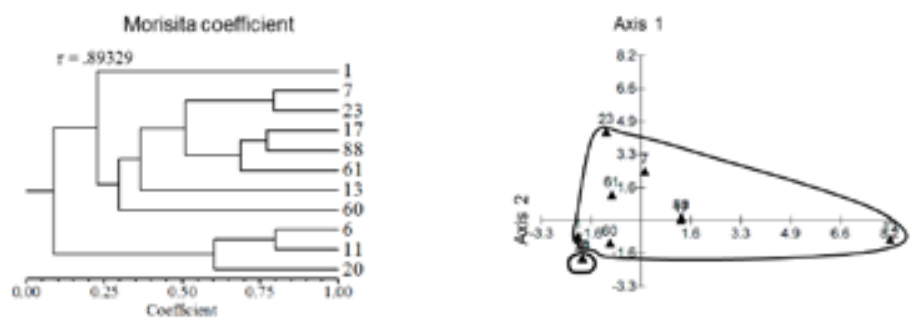

FIGURE 3

Similarities among the Complex A offerings using presence/absence and abundance data: cluster and PCA analysis.

was deposited in the fill. The opposite is indicated by the dominance index $(D)$ : higher values in the bilateral offerings and lower in the others (except again for Offering 6).

Sawfish (Pristis) is the common species in the bilateral axis offerings; in addition, the great quantity of halfbeaks found at Offering 13 is remarkable as is the great diversity of needlefish and halfbeaks in offering 17 (Table 3). In the case of the four offerings dedicated to Huitzilopochtli, despite the large number of species and individuals, the only fish found in all of them is sawfish, because Offering 6 lacks of any other type of fish; the remaining offerings, placed in ashlar stone boxes, share more species and genera (Table 3).
Finally, the three offerings dedicated to Tláloc have nine species in common, including sawfish (Table 3).

The correlation of the offerings placement to the above mentioned indices plus richness and abundance corroborate the empirical observations as well: 1) the richness and diversity of the offerings are linked to their spatial location (the bilateral axis vs. the shrines of the gods), and 2) all the ecological parameters are related to the type of container (fill vs. ashlar stone boxes: as a subset, the offerings in the fill have lower values of richness, abundance, diversity and equitability, and a higher value of dominance) (Table 4). 


\begin{tabular}{|c|c|c|c|c|c|c|c|c|c|c|c|c|c|}
\hline \multirow[b]{2}{*}{ Taxa } & \multirow[b]{2}{*}{ Offering } & \multicolumn{4}{|c|}{ Huitzilopochtli Side } & \multicolumn{4}{|c|}{ Bilateral Axis } & \multicolumn{3}{|c|}{ Tláloc Side } & \multirow[b]{2}{*}{ Total } \\
\hline & & 1 & 6 & 7 & 60 & 11 & 13 & 17 & 20 & 23 & 61 & 88 & \\
\hline \multicolumn{14}{|c|}{ FISH CONTENT } \\
\hline Carch & & - & - & - & 7 & - & - & - & - & 2 & - & 1 & 10 \\
\hline Carch & & - & - & - & - & - & 1 & 2 & - & - & 1 & 1 & 5 \\
\hline Carch & & - & - & - & - & - & - & 1 & - & - & - & - & 1 \\
\hline Galeo & & - & - & - & 1 & - & - & - & - & - & - & - & 1 \\
\hline Negal & & - & - & - & - & - & - & - & - & 1 & - & - & 1 \\
\hline Negal & & - & - & - & - & - & - & - & 1 & - & - & - & 1 \\
\hline Sphyr & & - & - & - & - & - & - & - & - & - & - & 1 & 1 \\
\hline Pristi & & - & 2 & 1 & 1 & 1 & - & 2 & 1 & 2 & 2 & 5 & 17 \\
\hline Pristi & & 1 & 1 & 2 & 2 & 1 & 1 & 2 & 2 & - & - & - & 12 \\
\hline Dasya & & - & - & - & - & - & - & - & - & - & 1 & 1 & 2 \\
\hline Hima & & - & - & - & 1 & - & - & - & - & 1 & - & - & 2 \\
\hline Dasya & ndet. & 1 & - & 1 & - & 1 & - & - & - & - & - & - & 3 \\
\hline Aetob & & - & - & - & - & - & - & - & 1 & - & - & - & 1 \\
\hline Clupe & & - & - & 1 & - & - & - & - & - & - & - & - & 1 \\
\hline Opsar & & - & - & 1 & - & - & - & - & - & 1 & - & 1 & 3 \\
\hline Ogco & & - & - & - & 3 & - & - & - & - & - & - & - & 3 \\
\hline Ablen & & - & - & 1 & - & - & - & - & - & - & - & - & 1 \\
\hline Stron & & - & - & 1 & - & - & - & 1 & - & - & - & - & 2 \\
\hline Stron & & - & - & - & - & - & - & 1 & - & - & - & - & 1 \\
\hline Stron & & - & - & 1 & 3 & - & - & 5 & - & - & - & - & 9 \\
\hline Tylost & & - & - & 5 & 1 & - & 4 & 11 & - & 9 & 12 & 6 & 48 \\
\hline Exocc & indet. & - & - & 1 & - & - & - & 1 & - & 4 & 2 & 1 & 9 \\
\hline Hemi & & 1 & - & 3 & 1 & - & 1 & 1 & - & 3 & 2 & 3 & 15 \\
\hline Hуро & & - & - & 7 & 3 & - & 32 & 9 & - & 3 & 2 & 10 & 66 \\
\hline Holoc & & 1 & - & - & - & - & - & - & - & - & - & - & 1 \\
\hline Fistul & & - & - & - & - & - & - & - & - & - & 1 & - & 1 \\
\hline Dacty & & - & - & 1 & - & - & - & - & - & - & - & 1 & 2 \\
\hline Scorp & & 1 & - & - & - & - & 1 & - & - & - & - & - & 2 \\
\hline Prion & & - & - & - & - & - & - & - & - & 4 & - & - & 4 \\
\hline Prion & & - & - & - & 1 & - & 1 & - & - & - & - & - & 2 \\
\hline Epine & ensionis & 3 & - & 1 & - & - & - & - & - & 4 & - & - & 8 \\
\hline Epine & & 1 & - & - & - & - & - & - & - & - & - & - & 1 \\
\hline Carar & & - & - & 1 & - & - & - & - & - & - & 2 & - & 3 \\
\hline Oligo & & - & - & 3 & - & - & - & - & - & - & - & - & 3 \\
\hline Selen & & - & - & - & - & - & - & - & - & 2 & - & - & 2 \\
\hline Lutja & entris & - & - & - & - & - & - & - & - & 2 & - & - & 2 \\
\hline Lutja & & - & - & - & - & - & - & - & - & - & 2 & - & 2 \\
\hline Lutja & L.Griseus & - & - & 1 & - & - & - & - & - & - & - & - & 1 \\
\hline Lutjal & & - & - & - & - & - & - & - & - & - & 1 & - & 1 \\
\hline Ocyu & & - & - & 2 & - & - & - & - & - & - & - & - & 2 \\
\hline Lobot & & - & - & - & - & - & 1 & - & - & - & - & - & 1 \\
\hline Aniso & & - & - & - & - & - & - & - & - & 1 & - & - & 1 \\
\hline Aniso & & 2 & - & 5 & - & - & - & - & - & 4 & 1 & - & 12 \\
\hline Haem & narium & - & - & 1 & - & - & - & - & - & - & - & - & 1 \\
\hline Haem & & - & - & 1 & - & - & - & - & - & 1 & 1 & - & 3 \\
\hline Poma & & 4 & - & 4 & 1 & - & - & - & - & 6 & 3 & 4 & 22 \\
\hline Kyph & & - & - & 1 & - & - & - & - & - & - & 1 & - & 2 \\
\hline Micro & & - & - & - & - & - & - & - & - & 1 & - & - & 1 \\
\hline Abude & & - & - & 1 & - & - & - & - & - & - & - & - & 1 \\
\hline Bodia & & 2 & - & 11 & - & - & - & - & - & 11 & 2 & 1 & 27 \\
\hline Halic & & - & - & 1 & - & - & - & - & - & 3 & 1 & - & 5 \\
\hline Scaru & & - & - & - & 1 & - & - & - & - & - & - & - & 1 \\
\hline
\end{tabular}




\begin{tabular}{|c|c|c|c|c|c|c|c|c|c|c|c|c|}
\hline Scarus vetula & 1 & - & - & - & - & - & - & - & - & - & - & 1 \\
\hline Sparisoma cf. S. Aurofrenatum & - & - & - & - & - & - & - & - & - & 1 & - & 1 \\
\hline Sparisoma rubripinne & - & - & 1 & - & - & - & - & - & 2 & - & - & 3 \\
\hline Sparisoma viride & 1 & - & - & - & - & - & - & - & 1 & - & - & 2 \\
\hline Chaetodipterus faber & - & - & - & 1 & - & - & - & - & - & - & - & 1 \\
\hline Acanthurus sp. & - & - & - & - & - & - & - & - & 1 & - & 1 & 2 \\
\hline Prionurus punctatus & - & - & - & - & - & - & - & - & 1 & - & - & 1 \\
\hline Sphyraena barracuda & - & - & 1 & - & - & - & - & - & 4 & - & - & 5 \\
\hline Trichiurus lepturus & - & - & - & - & - & - & - & - & 1 & - & - & 1 \\
\hline Canthidermis sufflamen & 1 & - & - & - & - & - & - & - & 1 & 2 & 1 & 5 \\
\hline Cantherhines sp. & - & - & - & - & - & - & - & - & 1 & - & - & 1 \\
\hline Aluterus sp. & - & - & 1 & - & - & - & - & - & - & - & - & 1 \\
\hline Acanthostracion sp. & - & - & - & 1 & - & - & - & - & - & - & - & 1 \\
\hline Lactophrys triqueter & - & - & - & - & - & - & - & - & 1 & - & - & 1 \\
\hline Ostraciidae gen.et $s p$. indet. & - & - & - & - & - & - & - & 1 & - & - & - & 1 \\
\hline Arothron sp. & 1 & - & - & - & - & - & - & - & 1 & - & - & 2 \\
\hline Sphoeroides annulatus & - & - & - & 1 & - & - & - & - & - & - & - & 1 \\
\hline Chilomycterus schoepfii & - & - & 1 & 1 & - & - & - & - & 2 & - & - & 4 \\
\hline Diodon holacanthus & - & - & - & 3 & - & - & - & - & 2 & - & - & 5 \\
\hline Diodon hystrix & - & - & 2 & 6 & - & 2 & - & - & 6 & 2 & 2 & 20 \\
\hline Diodon sp. & - & - & - & - & - & - & - & - & 3 & - & - & 3 \\
\hline a) individuals & 21 & 3 & 65 & 39 & 3 & 44 & 36 & 6 & 92 & 42 & 40 & 391 \\
\hline b) species & 13 & 2 & 30 & 18 & 2 & 9 & 9 & 4 & 33 & 20 & 16 & 63 \\
\hline ECOLOGICAL INDICES & & & & & & & & & & & & \\
\hline$D$ (Dominance) & .10 & .56 & .07 & .09 & .33 & .54 & .19 & .22 & .05 & .11 & .13 & - \\
\hline$H^{\prime}$ (Shannon-Wierner) & 2.49 & .64 & 3.06 & 2.67 & 1.1 & 1.11 & 1.96 & 1.56 & 3.24 & 2.65 & 2.39 & - \\
\hline$J^{\prime}$ (Equitability) & .94 & .92 & .89 & .91 & 1 & 0.5 & .82 & .97 & .92 & .89 & .86 & - \\
\hline
\end{tabular}

TABLE 3

Fish Species Comparison among Complex A Offerings (Species, MNI, and Diversity Indices).

\begin{tabular}{lccccccccccc}
\hline \multicolumn{1}{c}{ Attribute } & \multicolumn{2}{c}{ Richness } & \multicolumn{2}{c}{ Abundance } & \multicolumn{2}{c}{ Equitability } & \multicolumn{2}{c}{ Dominance } & \multicolumn{2}{c}{ Diversity } \\
& $r$ & $\mathrm{p}$ & $r$ & $\mathrm{p}$ & $r$ & $\mathrm{p}$ & $\mathrm{r}$ & $\mathrm{p}$ & $r$ & $\mathrm{p}$ \\
Spatial location & .68865 & .01911 & .48267 & .13265 & -.13484 & .69263 & -.58752 & .057351 & .62123 & .04134 \\
Vertical location & .51962 & .10138 & .34720 & .29549 & -.57735 & .06290 & -.40415 & .21766 & .46188 & .15266 \\
Horizontal location & -.26005 & .43996 & -.25099 & .45661 & .30821 & .35648 & .09631 & .77817 & -.16373 & .63048 \\
Container & .77460 & .00512 & .77636 & .00495 & -.71005 & .01436 & -.64550 & .03194 & .71005 & .01436 \\
\hline
\end{tabular}

Ranges established for the characteristics of the offerings:

$\begin{array}{lllll}\text { Spatial location: } & 0 \text { bilateral axis } & & 1 \text { Huitzilopochtli } & 2 \text { Tláloc } \\ \text { Vertical location: } & 0 \text { platform } & 1 \text { floor } & \\ \text { Horizontal location: } & 1 \mathrm{~N} \quad 2 \mathrm{~S} & 3 \mathrm{E} \quad 4 \mathrm{O} & 5 \mathrm{SE} \\ \text { Container: } & 1 \text { ashlar box } & 0 \text { underfloor fill } & \end{array}$

TABLE 4

Correlation of Offerings' Fish Communities to Location and Type of Container. Significant correlations are shaded ( $r>.6$, p $\leq .05)$. $r=$ Spearman's rho, $\mathrm{p}=$ probability.

The similarity of offerings using presence/absence data and the simple matching coefficient (Figure 3a) showed a very close relationship between the offerings deposited in the constructive fill (Offerings 6, 11, and 20). Their high similarity is based on the number of shared presences, but especially on the high number of shared absences. On the other hand, Offerings 7 and 23 are the most dissimilar as a result of their richness and the few species shared with the other offerings. Jaccard's 
coefficient emphasizes shared presences and shows two clear groups: one constituted by the three poorest offerings and the other by all of the other offerings. This last is the group for which dendrogram topography presents the greatest changes compared to the previous dendrogram. In particular, the increase in distance indicates that the similarity within each group is not as narrow as it seemed. Regardless of the coefficient used, at least three similar pairs of offerings are formed (Offerings 6-11, Offerings 13-17, and Offerings 61-88, besides Offerings 7-23 with Jaccard coefficient). Of these, only the pair formed by Offerings 13 and 17 coincides in having an opposite location on a main axis of symmetry.

The PCA analysis obtained for presence/absence data, confirmed the existence of two main clusters: one, disperse, composed by the richest and most dissimilar offerings $(7,23,60,61,88)$ and the other, compact, consisting of the less diverse offerings laid down in the fill, plus Offerings 13 and 17 (Figure 3a).

Similarity including abundance (Figure 3b) shows the formation of the two main groups observed with the Jaccard coefficient, although the internal arrangement differs in the group of the richest offerings, as well as the shorter similarity distances among them. This means that both sets are more similar in terms of the number of individuals involved for each species. Three pairs of most similar offerings are formed, but only two are common to those obtained in Jaccard's dendrogram (6-11 and 7-23), and no pair is located in the opposite position. Spatial relationships obtained with PCA show a high dispersion of the richest set of offerings deposited in cists, especially in Offering 13 (because of its high abundance of halfbeaks); as well as the great proximity of the three poorest offerings deposited in the fill, to one another.

\section{Comparison with ethnohistorical sources}

Ethnohistorical information on marine fishes in the Mexica culture is very scarce (Guzmán \& Polaco, 2003). Apparently, Mexica conquered a portion of the coast of the Gulf of Mexico during the rule of Motecuzoma Ilhuicamina, as a consequence for the deaths of the messengers who had gone to the coast to get fish and other marine organisms to serve as offerings to Huitzilopochtli, whose temple had been enlarged by that time; after conquering that region, the Mexicas received barbecued fish as a food tribute (Alvarado Tezozómoc, 1980). It is likely that the fishes in the Complex A offerings belonged this period of expansion and that they came from Veracruz, between Cotaxta and Zempoala. Although expert fishermen in the lakes of the basin of Mexico, it is unlikely it was the Mexicas who caught the marine fish in the offerings. Unfortunately, due to the shortage of documentary evidence, it is not possible to explain the presence in the offerings of fish that come from the Pacific Ocean based on documents.

The sources explicitly register the use of fish in two of the many Mexica rituals and festivities: one was during Izcalli, the last month of the civil calendar, the month of resurrection, suitable to renovate buildings. During that period, fishing and hunting were practiced, although the sources provide different versions about what happened with the hunted animals. They do not report the provenance of the animals either (Torquemada, 1986; Clavijero, 1987; Sahagún, 1992). The second festivity honored Uixtocíhuatl, the goddess of salt, in which sometimes a sawfish rostrum was used over the female victim's throat to prevent her from screaming (Sahagún, 1992). The final destination of the rostrum was not mentioned, but it might have been left as part of an offering.

Fray Bernardino de Sahagún was one of the chroniclers who most widely wrote about the customs of the Mexica people, and his works are of special interest because he interviewed Mexica wise old men approximately 50 years after the Spanish conquest. Of the 10 marine fish mentioned by this author, seven are among the most abundant and common fish in the offerings. Their persistence in the oral tradition was probably due to their frequent use in temple as offerings (Figure 4).

The review carried out on many ethnohistorical sources does not allow us to consider it possible to find additional information about the fishes and its cultural role. Thus, it is very important to recover fish remains appropriately and to study them as a primary and almost exclusive source of information about the relationships between Mesoamerican man and this resource. 

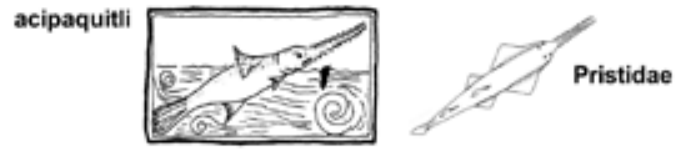

chimalmichi
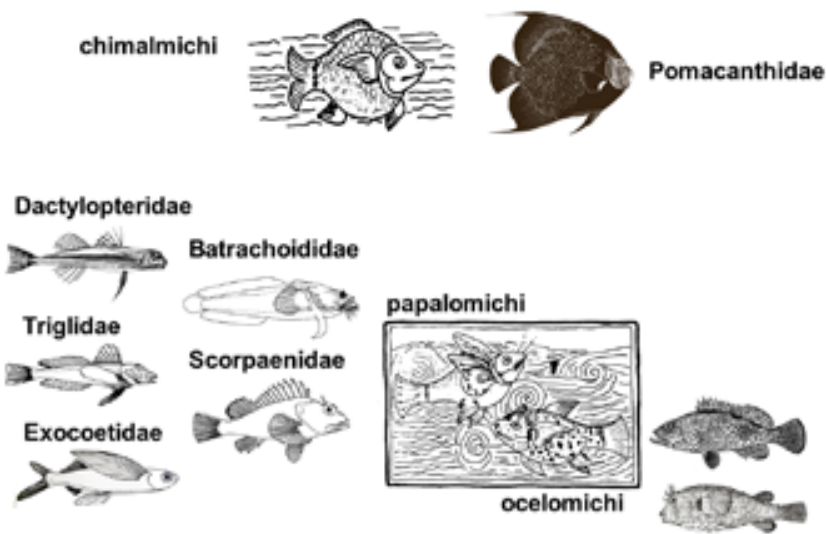

Serranidae

ocelomich

Diodontidae

quauhxouili
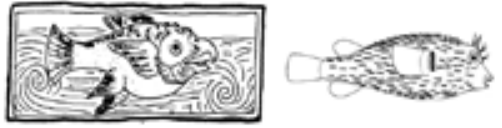

Diodontidae

totomichi
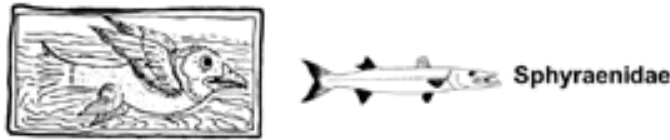

uitzitzilmichi

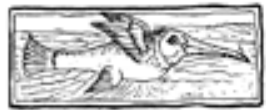

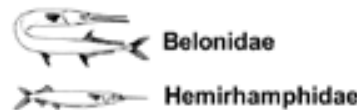

$36,+\infty$ Hemirhamphidae

FIGURE 4

Fishes portrayed in the Florentine Codex representing species in the Complex A offerings. Modified from Guzmán \& Polaco (2003). Pre-hispanic figures taken from López Luján (1993); modern figures taken from Carpenter (2002) and SIC (1976).

\section{CONCLUSIONS}

The study of the Complex A offerings shows several behavioral practices used by the Mexicas when constructing each offering's message: 1) the decision to use a large number of marine species, many of which are not usually found in domestic contexts or in other archaeological sites, emphasizing their ceremonial use, 2) excluding from the offerings many marine and freshwater groups common in domestic contexts -even the typical fishes of the region in which Tenochtitlan was settled-, 3) the widespread use, both in quantity and frequency, of $10 \%$ of the species, especially sawfish (Pristis) and which may be equivalent to the basic particles of the language of consecration and protection of the Templo Mayor, 4) a less discriminate use of most of the species, with which the message of oblation could have been particularized, 5) the use of taxidermically prepared fish indicating the existence of techniques to handle the bodies of animals, not necessarily mastered by the Mexica people but by the inhabitants of the place where the fish originated, 6) the message at the bilateral axis offerings differ more (are less diverse) than the offerings from other areas of the building, 
a difference further emphasized when the contents of the ashlar stone boxes and fill's offerings are compared, 7) there was no clear intention to make opposite offerings similar in fish content, except perhaps in offerings located in the bilateral axis of the building.

\section{ACKNOWLEDGMENTS}

Elizabeth Reitz read an English draft of the text and helped with her comments to improve the writing and content.

\section{REFERENCES}

Allen, G.R. \& Robertson, D.R. 1994: Fishes of the tropical eastern Pacific. University of Hawaii Press, Honolulu, Hawaii.

Alvarado Tezozómoc, H. 1980: Crónica mexicana. $3^{\text {rd }}$ ed. Porrúa, México.

Bedia Sánchez, C. \& Franco López, J. 2008: Peces de los sistemas costeros del estado de Veracruz. FES Iztacala, Universidad Nacional Autónoma de México, México.

CARPEnTER, K.E. (ed.) 2002: The living marine resources of the Western Central Atlantic. Volumes 1-3. FAO Species Identification Guide for Fishery Purposes and American Society of Ichthyologists and Herpetologists Special Publication No. 5. Rome, FAO.

CARRAmiÑAnA A., E. 1988: Informe preliminar sobre la ofrenda zoológica dedicada a Coyolxauhqui. Antropología 3: 225-250.

Carricart-Ganivet, J.P. \& Horta-Puga, G. 1993: Arrecifes de coral en México. In: Salazar-Vallejo, S.I. \& González, N.E. (eds.): Biodiversidad marina y costera de México: 80-90. Comisión Nacional para el Conocimiento y Uso de la Biodiversidad y Centro de Investigaciones de Quintana Roo, México.

Castro-Aguirre, J.L.; Espinosa Pérez, H.S. \& SchmiTTER-Soto, J.J. 1999: Ictiofauna estuarino-lagunar y vicaria de México. Instituto Politécnico Nacional, Editorial Limusa y Noriega Editores, México.

Clavijero, F.J. 1987: Historia antigua de México. Porrúa, México.

Crisci, J.V. \& López Armengol, M.F. 1983: Introducción a la teoría y práctica de la taxonomía numérica. Programa Regional de Desarrollo Científico y Tecno- lógico, Secretaría General de la Organización de los Estados Americanos, Washington.

DíAZ-PARdo, E. 1982: Restos de peces procedentes de la ofrenda 7. In: Matos Moctezuma, M. (ed.): El Templo Mayor: excavaciones y estudios: 151-160. Instituto Nacional de Antropología e Historia, México.

Díaz-Pardo, E. \& Teniente-Nivón, E. 1991: Aspectos biológicos y ecológicos de la ictiofauna rescatada en el Templo Mayor, México. In: Polaco, O.J. (coord.): La fauna en el Templo Mayor: 33-104. Asociación de Amigos del Templo Mayor, Instituto Nacional de Antropología e Historia, and García y Valadés editores, México.

García Cook, Á. \& Arana A.R.M. 1978: Rescate arqueológico del monolito Coyolxauhqui: informe preliminar. Instituto Nacional de Antropología e Historia, México.

GuZmán, A.F. 2007: Los peces de las ofrendas del Complejo A del Templo Mayor: una aproximación biológica y arqueozoológica. Facultad de Ciencias, Universidad Autónoma de Madrid, Madrid.

Guzmán, A.F. \& Polaco, O.J. 1999: La Ofrenda 23 del Templo Mayor de Tenochtitlan vista a través de los peces. Arqueología 22: 115-124.

- 2000: Los peces arqueológicos de la Ofrenda 23 del Templo Mayor de Tenochtitlan. Instituto Nacional de Antropología e Historia, México.

- 2003: A comparative analysis of fish remains from some Mexica offerings. Archaeofauna 12: 7-20.

- 2007: Fishing in Pre-hispanic México. In: Hüster Plogmann, H. (ed.): The role of fishing in ancient time: proceedings of the $13^{\text {th }}$ Meeting of the ICAZ-Fish Remains Working Group Meeting: 25-36. Internationale Archäologie, Arbeitsgemeinshcalt, Symposium, Tagung, Kongress, Band 8. Verlag Marie Leidorf GmbH. Rahden, Westf.

Humann, P. 1994: Reef fish identification: Florida-Caribbean-Bahamas. $2^{\text {nd }}$ ed., New World Publications, Jacksonville, Florida.

Kennett, D.J.; Voorhies, B.; Wake, T.A. \& Martínez, N. 2008: Long-term effects of human predation on marine ecosystems in Guerrero, México. In: Rick, T.C. \& Erlandson, J.M. (eds.): Human impacts on ancient marine ecosystems: a global perspective: 103-124. University of California Press, Berkeley.

KleIN, R.G. \& CRUZ-Uribe, K. 1984: The analysis of animal bones from archaeological sites. The University of Chicago Press, Illinois.

LóPEz LujÁn, L. 1993: Peces y moluscos en el libro undécimo del Códice Florentino. In: Polaco, O.J. (coord.): La fauna en el Templo Mayor: 213-263. Asociación de Amigos del Templo Mayor, Instituto Nacional de 
Antropología e Historia, and García y Valadés editores, México.

López Luján, L. \& Chávez Balderas, X. 2010: Al pie del Templo Mayor: excavaciones en busca de los soberanos mexicas. In: López Luján, L. \& McEwan, C. (coord.): Moctezuma II: tiempo y destino de un gobernante, México: 294-303. Instituto Nacional de Antropología e Historia/The British Museum, México.

Matos Moctezuma, E. 1990: El proyecto Templo Mayor: objetivos y programas. In: Matos Moctezuma, E. (coord.): Trabajos arqueológicos en el centro de la Ciudad de México: 17-39. Instituto Nacional de Antropología e Historia, México.

Polaco, O.J. 1991: La fauna en el Templo Mayor: una aproximación metodológica. In: Polaco, O.J. (coord.): La fauna en el Templo Mayor: 15-31. Asociación de Amigos del Templo Mayor, Instituto Nacional de Antropología e Historia, and García y Valadés editores, México.

Polaco, O.J. \& Guzmán, A.F. 1997: Arqueoictiofauna mexicana. Instituto Nacional de Antropología e Historia, México.

Reitz, E.J. \& Wing, E.S. 1999: Zooarchaeology. Cambridge Manuals in Archaeology. Cambridge University Press, Cambridge.
Reyes Bonilla, H. 1993: Biogeografía y ecología de los corales hermatípicos (Anthozoa: Scleractinia) del Pacífico de México. In: Salazar-Vallejo, S.I. \& González, N.E. (eds.): Biodiversidad marina y costera de México: 207-222. Comisión Nacional para el Conocimiento y Uso de la Biodiversidad y Centro de Investigaciones de Quintana Roo, México.

Rodríguez Galicia, B. \& Valadez Azúa, R. 2013: Vestigios del recurso costero en el sitio arqueológico de Teopancazco, Teotihuacan. Estado de México. Revista Española de Antropología Americana 43(1): 9-29.

SaHagún, B. de 1992: Historia general de las cosas de Nueva España. $8^{\text {th }}$ ed. Porrúa, México.

SEviLLA, M.L. 1977: Introducción a la ecología marina. Instituto Politécnico Nacional, México.

SIC. 1976. Catálogo de peces marinos mexicanos. Secretaría de Industria y Comercio, México.

Sмith, R.L. \& Sмiтh, T.M. 2004: Ecología. $4^{\text {th }}$ ed. Pearson/Addison Wesley, Madrid.

Torquemada, J. de 1986: Monarquía indiana. Vol. II. Porrúa, México. 
